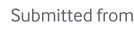 BMJ Quality
}

\section{A project to reduce the rate of central line associated bloodstream infection in ICU patients to a target of zero}

\author{
Muhammad Yaseen, Fahad Al-Hameed, Khalid Osman, Mansour Al-Janadi, \\ Majid Al-Shamrani, Asim Al-Saedi, Abdulhakeem Al-Thaqafi
}

To cite: Yaseen M, AlHameed F, Osman K, et al. A project to reduce the rate of central line associated bloodstream infection in ICU patients to a target of zero. BMJ Quality Improvement Reports 2016;5:u212545. w4986. doi:10.1136/ bmjquality.u212545.w4986

Received 26 April 2016 Revised 31 May 2016 Published Online First 11 August 2016
CrossMark

King Abdulaziz Medical City; National Guard Health Affairs, Jeddah, Saudi Arabia

Correspondence to Muhammad Yaseen yaseenmf@ngha.med.sa

\section{ABSTRACT}

Central venous catheters (CVCs) are life-saving and the majority of patients in intensive care units (ICUs) have them placed in order to receive medicine and fluids. However, the use of these catheters can result in serious bloodstream infections. The rate of Central Line Associated Blood Stream Infection (CLABSI) in Adult Intensive Care Units (ICUs) at King Abdulaziz Medical City Jeddah (KAMC-J) at the start of the project was 2.0/1000 line days in 2008. The Central Line (CL) Bundle by the Institute of Healthcare Improvement (IHI) was implemented at the same time with monitoring of compliance to the CL Bundle. The compliance to CL Bundle was very low at $37 \%$ in the same period. A multidisciplinary team was created to improve the compliance to the CL bundle which was expected to have an impact on the rate of CLABSI to achieve zero CLABSI events. The team continued to monitor and evaluate the progress on the compliance to the bundle as well as monitoring the CLABSI events using National Healthcare Safety Network diagnostic criteria. The real reduction in the rate of CLABSI was achieved in 2010 with $0.7 / 1,000$ device days when the compliance to CL Bundle reached up to $98 \%$ in that year and $100 \%$ in the next two subsequent years. The project still continued and the rate continued to drop and the ultimate target of zero CLABSI was achieved in the year 2014 and maintained in the year 2015 with a sustained compliance of $100 \%$ to the CL Bundle. Successful implementation of CL Bundle can help in reducing the rates of CLABSI and achieving zero CLABSI events for a sustained period.

\section{PROBLEM}

Central venous catheters are life-saving and the majority of patients in intensive care units (ICUs) required them for medicine and fluids resuscitations. However, the use of these catheters can result in serious bloodstream infections. ${ }^{1}$ These bloodstream infections could result in increased mortality, morbidity and hospital length of stay. ${ }^{2-5}$

King Abdulaziz Medical City Jeddah is an over 600 bed tertiary care hospital serving the National Guards and their eligible dependents in the Western Region of Saudi Arabia. Adult ICU is a combined unit of medical and surgical patients with a bed capacity of 28 beds. The CLABSI (Central Line Associated Bloodstream Infection) rate in Adult intensive care units at King Abdulaziz Medical City Jeddah was averaging 2.0 per 1000 device days in 2008. Despite individual measures and efforts, the rate of CLABSI remained a cause of concern. The infection control and adult intensive care teams considered this an area for improvement and looked for measures to resolve this issue. A multidisciplinary CLABSI team was created with an aim to improve compliance with the CL bundle, and subsequently reduce CLABSI rates. Our population included all Adult ICU patients who required a central line insertion for treatment. CLABSI was defined according to the criteria of GCC Center for Infection Control Surveillance Manual. ${ }^{6}$

\section{BACKGROUND}

Evidence based clinical practice guidelines have recommended multiple strategies to reduce CLABSI rate. Despite this, CLABSI remains a significant cause of mortality and morbidity in patients who have a central line in situ worldwide. ${ }^{2-5}$ Acquiring CLABSI is associated with prolonged usage of central lines and increased ICU and hospital length of stay, ultimately costing more than US $\$ 40,000$ per episode. $^{78}$ All regulatory bodies and infection prevention organizations emphasize the importance of reducing hospital acquired infections, and have published guidelines of practice recommendations to address them, notably the Central line bundle by the Institute of Healthcare Improvement (IHI). ${ }^{9}$ This IHI CL bundle, a grouping of best practices that, when applied together, may result in substantially greater improvement, has been implemented in 
many ICU, along with teamwork and communication strategies such as structured multidisciplinary rounds and daily goal setting, to achieve the goal of zero CLABSI for a sustainable time. ${ }^{10} 11$

At King Abdulaziz Medical City Jeddah, a multidisciplinary team was created in Adult ICU with the aim to achieve an ultimate goal of zero CLABSI events for a sustained period. The team worked on CLABSI prevention strategies including the implementation of Central Line bundle with a target of at least $90 \%$ compliance. Two champions were identified from the intensive care and from infection control departments who lead this important quality project.

\section{BASELINE MEASUREMENT}

The data was collected by the Infection Control Practitioners on the events of CLABSI and it was diagnosed based on the definition criteria set by National Healthcare Safety Network of the US Center for Disease Control and Prevention. The overall compliance to $\mathrm{CL}$ Bundle was considered zero even if only one of the five elements was not compliant. CLABSI rate per 1000 device days. Our baseline CLABSI rate at the start of the project in the first quarter of 2008 was 2.8/1000 device days and the average rate for the year 2008 was 2.0/1000 line days. The compliance rate to the central line bundle was $37 \%$ in 2008 . The compliance was measured as the number of intensive care unit patients with a central line, for whom all four elements of the central line bundle are documented and in place divided by the total number of intensive care unit patients with central line.

\section{DESIGN}

In January 2008, a Multidisciplinary team at King Abdulaziz Medical City was created to work on achieving and sustaining the target of zero CLABSI events. The team adopted the IHI (Institute of Healthcare Improvement) Central Line Bundle to reduce the rate of CLABSI in Adult ICU. The Bundle elements included: Hand hygiene, Maximal barrier precautions, Chlorhexidine skin antisepsis, Optimal catheter site selection with subclavian vein as the preferred site for insertion, and Daily review of line necessity.

The infection control practitioners monitored the events of CLABSI and the compliance to central line bundle. A team of nurses was trained using the "train the trainer" approach to monitor and complete the insertion part of the central line bundle. The data was analyzed quarterly and presented to the concerned teams as well as the Infection Control Committee. The FOCUS PDSA cycle was carried out with corrective actions until desirable results were achieved.

\section{STRATEGY}

The multidisciplinary team included Intensivist as the co-leader of the team along with the Director of
Chart 1 CLABSI rate in Adult ICU of KAMC-J from 2008-2015

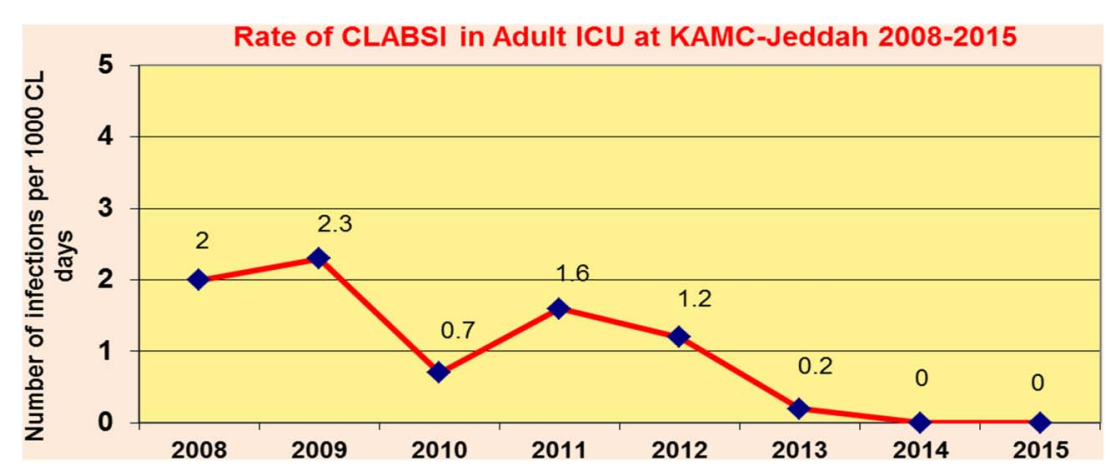

Chart 2 Compliance to Central Line Bundle in Adult ICUs at KAMC-J from 2008-2015

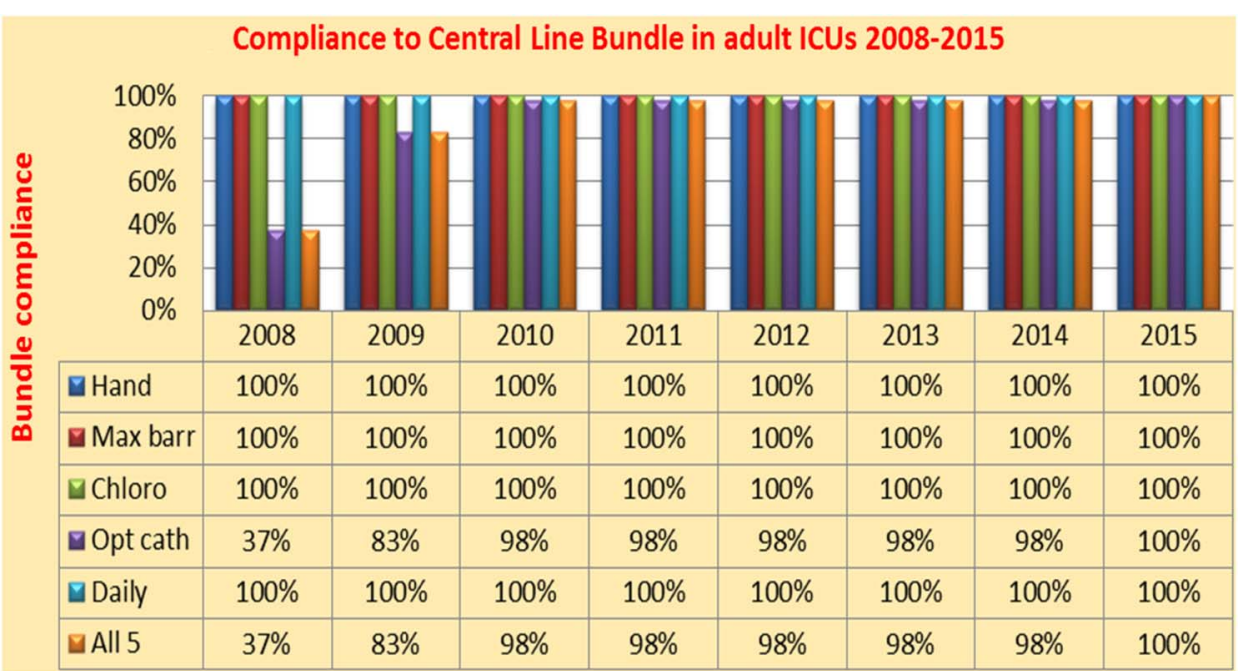


Table 1. Central line days and CLABSI Events in Adult ICUs at KAMC-J from 2008-2015

\begin{tabular}{llll}
\hline & $\begin{array}{l}\text { Central } \\
\text { Line Days }\end{array}$ & $\begin{array}{l}\text { Number of } \\
\text { CLABSI } \\
\text { Events }\end{array}$ & $\begin{array}{l}\text { CLABSI Rate per } \\
\mathbf{1 0 0 0} \text { Line Days }\end{array}$ \\
\hline $\mathbf{2 0 0 8}$ & 458 & 2 & 4.4 \\
$\mathbf{2 0 0 9}$ & 659 & 2 & 3.0 \\
$\mathbf{2 0 1 0}$ & 557 & 5 & 9.0 \\
$\mathbf{2 0 1 1}$ & 600 & 1 & 1.7 \\
$\mathbf{2 0 1 2}$ & 950 & 2 & 2.1 \\
$\mathbf{2 0 1 3}$ & 4429 & 1 & 0.2 \\
$\mathbf{2 0 1 4}$ & 6270 & 0 & 0.0 \\
$\mathbf{2 0 1 5}$ & 3168 & 0 & 0.0 \\
\hline
\end{tabular}

Infection Prevention and Control and the Infection Control. The other members included: Infection Control Practitioner who acted as the coordinator of the team to organize team meetings, ICU team leader, ICU doctors, and ICU nurses.

The team adopted the IHI central line bundle to measure the practice of central line usage including the daily review of central line necessity. Educational sessions were conducted for all healthcare workers in the ICU especially nurses and physicians before implementation of the central line bundle. The educational sessions included the details of all elements of the bundle and the scientific evidence to show its effectiveness of preventing the central line infections. Special sessions were conducted for the nursing team leaders on monitoring the process of central line insertion and completing the central line bundle form. The logistics department was also advised to keep constant supply of Chlorhexidine containing swabs which are used for preparation of the central line insertion site. Preparation of insertion site with alcoholic chlorhxidine was one of the elements of the bundle.

The multidisciplinary CLABSI Elimination Team met regularly to review the CLABSI events and discuss any issues with implementation of central line bundle and compliance to its elements. Any new scientific evidence or research relevant to the project was disseminated amongst the team members. The FOCUS PDSA cycle continued with corrective actions until desirable results were achieved.

\section{RESULTS}

The insertion site was the most deficient element in the beginning but the compliance to this element improved greatly by the end of the project $(100 \%)$. A remarkable drop in CLABSI rate was noted, as the bundle compliance kept rising to an optimum $100 \%$. The rate of CLABSI in the first quarter of 2008 at the start of this project was 2.8/1,000 device days while the overall rate in the year 2008 was 2.0/1,000 device days. The real reduction in the rate of CLABSI was achieved in 2010 with $0.7 / 1,000$ device days when the compliance to Bundle reached up to $98 \%$ in that year and $100 \%$ in the next two subsequent years. The project still continued and the rate continued to drop and the ultimate target of zero CLABSI was achieved in the year 2014 and maintained throughout the year 2015. For the complete results, see Chart $1 \& 2$ and Table 1.

\section{LESSONS AND LIMITATIONS}

Several lessons we learned from this project; firstly, the greatest barrier to the implementation of the bundle was the reluctance of consultants to use of subclavian site for insertion of central lines but consistent feedback on the local rates of CLABSI and persistence of the team to convince them with scientific evidence eventually worked, and they changed their practice. So the lesson is that persistence pays at the end. Secondly, all of the stakeholders in the process must be included, in order to gain the buy-in and cooperation of all parties. In order to enlist support and engage staff, it was important to share the results with the stakeholders. The data was limited by the fact that this study is from a single center and only adult ICU patients were included.
Chart 3 Compliance to $\mathrm{CL}$ bundle vs. CLABSI Events in Adult ICUs at KAMC-J from 2008-2015

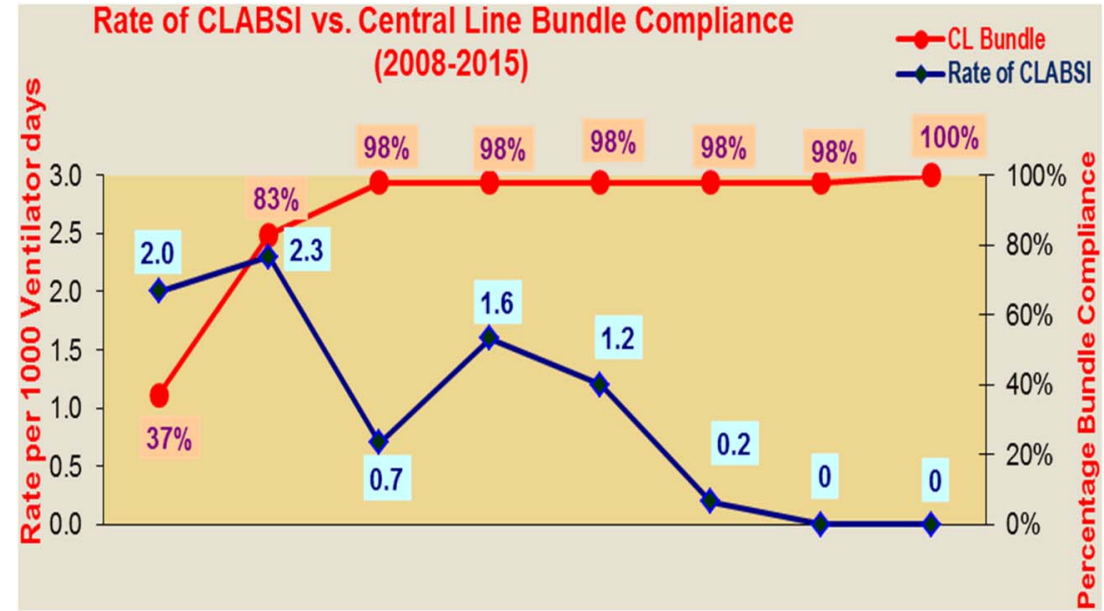




\section{CONCLUSION}

To support the success of the project, it should be endorsed by science, led by stakeholders, guided by measures, and supported by management. Implementing CL Bundle and maintaining excellent compliance is the key in having this excellent result in our study. The team had quite a few challenges in implementation and compliance to the bundles in the beginning but eventually, with the dedication of the whole team, desirable results were achieved. With the CLABSI rate of 2.0/1000 line days and $37 \%$ compliance to the CL bundle at the baseline, an ultimate goal of zero CLABSI for sustained two years and $100 \%$ compliance to the CL bundle was achieved. This also proves that the bundle compliance which is the measure of clinical practice is directly linked to the outcome in the form of CLABSI events as seen in chart 3. This is a great achievement and planning is being done to use the same model and interventions for reduction of CLABSI rates in other units especially pediatric ICU, and Hematology and Oncology units.

Acknowledgements Medhat Lamfon, Allan Catu, Areej Qudsi, Ang Seok Gor.

Declaration of interests Nothing to declare

Ethical approval According to the policy activities that constitute research at King Abdulaziz Medical City, this work met criteria for operational improvement activities exempt from ethics review. Local policies and procedure do not require IRB approval for improvement projects of that kind. The work is primarily intended to improve local care, not provide generalizable knowledge in a field of inquiry. Ethical approval was not obtained as this is considered to be a quality improvement project, where all central line bundle interventions were validated by evidence based research, and testing was performed solely for improvement purposes.

Open Access This is an open-access article distributed under the terms of the Creative Commons Attribution Non-commercial License, which permits use, distribution, and reproduction in any medium, provided the original work is properly cited, the use is non commercial and is otherwise in compliance with the license. See:

- $\mathrm{http}: / /$ creativecommons.org/licenses/by-nc/2.0/

- http://creativecommons.org/licenses/by-nc/2.0/legalcode

\section{REFERENCES}

1. Maki DG, Kluger DM, Crnich CJ. The risk of bloodstream infection in adults with different intravascular devices: a systematic review of 200 published prospective studies. Mayo Clin Proc. 2006;81:1159-1171.

2. Centers for Disease Control and Prevention (CDC): Vital signs: central line-associated bloodstream infection - United States, 2001 2008, and 2009. MMWR Morb Mortal Wkly Rep. 2011;60:243-248.

3. Fagan RP, Edwards JR, Park BJ, Fridkin SK, Magill SS. Incidence trends in pathogen-specific central line-associated bloodstream infections in US intensive care units. Infect Control Hosp Epidemiol. 2013;36:893-899.

4. Boyce JM. Prevention of central line-associated bloodstream infections in hemodialysis patients. Infect Control Hosp Epidemiol. 2012;33:936-944

5. Rosenthal VD. Central line-associated bloodstream infections in limited-resource countries: a review of the literature. Clin Infect Dis. 2009:49:1899-1907.

6. GCC Centre for Infection Control and National Guard HealthAffairs. Surveillance manual; 2012. http://ngha.med.sa/English/ MedicalCities/AlRiyadh/MedicalServices/Documents/ SurveillanceManual2012.pdf [accessed 24.04.16].

7. Rosenthal VD, Guzman S, Migone O, Crnich CJ. The attributable cost length of hospital stay, and mortality of central line-associated bloodstream infection in intensive care department in Argentina: a prospective, matched analysis. Am J Infect Control. 2003;34:475-480.

8. Higuera F, Rangel-Frausto MS, Rosenthal VD, et al. Attributable cost and length of stay for patients with central venous catheter venous catheter-associated bloodstream infection in Mexico City intensive care units: a prospective, matched analysis. Infect Control Hosp Epidemiol. 2007;28:31-35.

9. Institute for Healthcare Improvement. Implement the IHI Central Line Bundle. Accessed April 24, 2016.http://www.ihi.org/knowledge/ Pages/Changes/ImplementtheCentralLineBundle.aspx.

10. Pronovost $P$, Needham $D$, Berenholtz $S$, et al. An intervention to decrease catheter-related bloodstream infections in the ICU. N Engl $J$ Med. 2006;355:2725-2732.

11. Coopersmith CM, Rebmann TL, Zack JE, et al. Effect of an education program on decreasing catheter-related bloodstream infections in the surgical intensive care unit. Crit Care Med. 2002;30:59-64. 